\title{
Experimental Validation of an Ionospheric Monitoring Scheme for Dual Frequency GBAS
}

\author{
Michael Felux, Mihaela-Simona Circiu, Daniel Gerbeth, Maria Caamano \\ German Aerospace Center (DLR)
}

\begin{abstract}
In this paper we describe a method to monitor for a difference in the ionospheric delay observed by a ground station of a Ground Based Augmentation System (GBAS) and an airborne user. In case of detection the affected satellite can be excluded or a switch to an ionospheric free processing mode can be triggered. As it is not possible to estimate the absolute ionospheric delay at the ground station from the transmitted corrections directly, we compare a pseudo-ionospheric delay estimate from the corrections with an ionospheric estimate after removing biases. We then show the performance of the proposed monitoring architecture in flight trials from our GBAS test environment for different scenarios. In order to obtain results for a full constellation we considered an L1/L2 dual frequency combination where the expected noise and multipath is larger than in the L1/L5 case which will be used in an operational GBAS. Results show that even with the larger test statistic and in the single constellation case the monitor is feasible and provides good results. Furthermore, we also show results using the available L5/E5a signals collected during flight tests. Also in this case the performance is good and the threshold was not exceeded despite the low threshold of the monitor due to the marginal geometry. In order to test the reaction of the monitor to ionospheric gradients we injected a simulated error into the airborne measurements. As the monitoring threshold depends on the satellite geometry we show some exemplary results of the impact of different gradient slopes on the test statistic of the affected satellites.
\end{abstract}

\section{INTRODUCTION}

The Ground Based Augmentation System (GBAS) is a landing system for aircraft. A reference station, consisting of typically four reference receivers at carefully surveyed locations at an airport, provides corrections for navigation signals from Global Navigation Satellite Systems (GNSS). Along with these corrections integrity parameters are broadcast such that the airborne receivers can correct their GNSS measurements and calculate bounds to the residual position errors. Currently, there are ground stations commercially available and already in use at several locations including Bremen and Frankfurt (Germany), Newark and Houston (United States) Sydney (Australia), Malaga (Spain) and Zurich (Switzerland). These ground stations enable approaches under CAT-I weather conditions corresponding to the GBAS Approach Service Type (GAST) C in GBAS phraseology. Standards for GAST D (i.e. the service type supporting also CATII/III approaches) are in the final stage of development and will be included in ICAO's Standards and Recommended Practices (SARPS) at the end of 2016.

All those service types, however, rely only on the GPS constellation and signals in the L1 frequency band. Therefore, the measures to protect users against ionospheric disturbances in GAST C and D may result in limited availability, especially in equatorial regions with highly active ionospheric conditions.

Since the introduction of the latest generation of GPS satellites (Block IIF) and with the introduction of the European Galileo constellation a growing number of satellites offering navigation signals on a second usable frequency are becoming widely available. With these it is possible to form a dual-frequency combination of the measured pseudoranges to remove the significant part of the ionospheric delay and thus make GBAS robust against ionospheric gradients. Increasing the number of navigation satellites through the use of dual-constellation algorithms furthermore provides a significantly increased robustness against ionospheric scintillations and associated loss of lock events. As the protection against the ionospheric gradient threat requires a significant amount of monitoring or conservative error bounding one of the new processing modes of a future dual frequency dual constellation (DFDC) GBAS will be an ionospheric free (Ifree) combination of the ranging signals. This combination of signals from two different frequencies allows the removal of the ionospheric delay (except for higher order effects) and thus effectively eliminates the gradient threat. However, this comes at a cost of significantly increased residual noise in the position solution. The Ifree solution combines code measurement from two frequencies and thus also the residual errors contained in those measurements. It is thus very likely that the primary processing mode of a future GBAS will be a single frequency mode. In that case, however, a switching trigger from single frequency to Ifree positioning is necessary. In this paper we present a possibility of such a switching trigger as part of the airborne monitoring scheme. After a description of the background and the principles we show an experimental validation of the proposed method based on dual frequency flight test data. 


\section{FUTURE GBAS PROCESSING MODES}

To date there is no clear way defined how future dual frequency and multi constellation GBAS processing and integrity monitoring will be accomplished. Within the European Sesar program several different concepts are under investigation. The difficult goal is to enable new processing modes which overcome the challenges of current GAST C/D GBAS. At the same time it is mandatory to maintain backwards compatibility to those service types. Furthermore, the architecture needs to be simple to avoid too many different modes and thus keep the standardization and certification efforts as small as possible.

For backwards compatibility both GAST C and D modes are expected to be also supported in the future. Other modes likely to be supported include GAST C and D-like processing but using a second constellation in addition to GPS. This mode provides a larger number of usable satellites and thus in general significantly improved satellite geometries. This yields more robustness during ionospheric scintillation conditions because it is very likely that tracking of a sufficient number of satellites can be maintained. Note that during scintillation conditions an Ifree processing may not be desirable as the signals on both frequencies will have to be tracked simultaneously and thus the probability of losing a satellite is increased compared to single frequency processing modes.

In case the airborne receiver is capable of tracking L5/E5a signals as well, positioning can be performed in a single frequency way (either L1/E1 or L5/E5a), however, with dual frequency monitoring for ionospheric gradients. As we showed in previous studies the single frequency modes provide a better nominal performance than a dual frequency positioning [1]. In this configuration the better nominal performance can be kept maintained in most cases. Should an ionospheric gradient situation be detected by the dual frequency airborne monitoring a switch to an Ifree positioning can be performed.

Due to the very constrained capacity of the VHF data link and the required backwards compatibility the possibilities for the definition of new modes is limited. A detailed study about this issue concludes that it will probably only be possible to transmit one set of corrections for a new frequency [2]. It is thus essential that all GBAS modes can be supported by using only the existing GAST C/D corrections and integrity parameters and one set of additional corrections and integrity parameters. One way of achieving this goal is the provision of additional single frequency corrections for L5/E5a. With those it is possible to do either single frequency L5/E5a positioning or form Ifree corrections by combining the GAST C/D corrections (plus potentially the E1 corrections from a second constellation) and L5/E5a corrections [4][2].

\section{DUAL FREQUENCY MONITORING}

In order to protect a dual frequency user against the ionospheric gradient threat in the case of single frequency positioning a dedicated monitor for ionospheric gradients is required. The monitor should fulfill the following criteria:

1. Reliably detect ionospheric gradients between ground station and airborne user

2. Have a sufficiently low false alarm probability

3. Preferably be a range-based monitor to exclude only affected satellites

4. Be an airborne monitor such that no assumption about airborne performance has to be made by the ground station

5. Take into account the actual geometry used in order to avoid unnecessary conservatism

In order to detect a difference in the observed ionosphere at the ground station and a user ideally we would compare ionospheric delay estimates at the ground and at the user. However, due to the way the corrections are formed in the ground station, it is not possible to estimate the ionospheric delay from the transmitted corrections. In a process called "smoothed clock adjust" the delays common to all satellites are removed. The largest portion of this will be the receiver clock but it also includes the part of the ionospheric delay that is common to all satellites. For that reason, only the information about the difference in the ionospheric delay between the satellites is contained in the corrections [5].

An ionospheric delay common to all satellites does, however, not affect the positioning of the airborne user since the common part maps into the user clock estimate. We thus propose to compare a pseudo ionospheric delay from the corrections and the ionospheric delay from the airborne receiver after removing all biases to detect potential ionospheric gradients. All the following steps are carried out for a set of $N$ satellites that the airborne system uses for positioning and for which corrections from the ground system are available.

We start by estimating the pseudo-ionospheric delay $\tilde{I}_{P R C, i}$ on L1 from the corrections as

$$
\begin{array}{r}
\tilde{I}_{P R C, i}=\frac{f_{L 5}^{2}}{f_{L 1}^{2}-f_{L 5}^{2}} \cdot\left[\left(P R C_{L 5, i}+\Delta t \cdot R R C_{L 5, i}\right)-\right. \\
\left.\left(P R C_{L 1, i}+\Delta t \cdot R R C_{L 1, i}\right)\right]
\end{array}
$$

where $P R C_{f, i}$ and $R R C_{f, i}$ are the transmitted pseudorange and range rate corrections for satellite $i$ on frequency $f$, $\Delta t$ is the time difference between the current time and the time at which the corrections were generated.

From the airborne measurements we can form the ionospheric delay estimate $\tilde{I}_{a i r, i}$ for the delay on L1 in a similar way by using the smoothed airborne code measurements $\hat{\rho}_{L 5, i}$ and $\hat{\rho}_{L 1, i}$ as 


$$
\tilde{I}_{\text {air }, i}=\frac{f_{L 5}^{2}}{f_{L 1}^{2}-f_{L 5}^{2}}\left(\hat{\rho}_{L 5, i}-\hat{\rho}_{L 1, i}\right)
$$

Note that the airborne receiver operates at a higher update rate than the ground system. The time difference between the time of applicability of the corrections and the pseudorange measurements is accounted for in the calculation of $\tilde{I}_{P R C, i}$, as described in Equation (1). The two quantities $\tilde{I}_{P R C, i}$ and $\tilde{I}_{\text {air }, i}$ are, however, not yet comparable. First we have to account for the fact that the user clock estimate may be removed with a different set of satellites than the airborne receiver uses. For that reason we remove again the average of the pseudo ionospheric delay estimate from the corrections and obtain

$$
I_{P R C, i}=\tilde{I}_{P R C, i}-\frac{1}{N} \sum_{i=1}^{N} \tilde{I}_{\text {PRC, }}
$$

which we will use later on in forming the test statistic.

In order to make the airborne ionospheric delay estimates comparable we also remove the average of all ionospheric delay estimates from the same subset as used previously for the estimation of the pseudo iono delay from the corrections by forming the value

$$
I_{\text {air }, i}=\tilde{I}_{\text {air }, i}-\frac{1}{N} \sum_{i=1}^{N} \tilde{I}_{\text {air }, i}
$$

Those two quantities from Equations (3) and (4) are now comparable and are used to form the test statistic for the monitor as

$$
I_{\text {test }, i}=\left|I_{\text {air }, i}+I_{P R C, i}\right|
$$

As the iono estimate from the corrections has opposite sign due to the way the corrections are defined we do not subtract but add both values. In order to decide now if the difference of the observations from the ground and airborne system poses a threat to the airborne user or not a threshold for this monitor is necessary. In [5] we derived the monitoring threshold based on the airworthiness requirements as given in the CS-AWO [8] and the AC120 28B [9]. The derivation uses the requirement to land within the touchdown box on the runway. Depending on the autopilot performance a largest vertical position error can be determined for which the aircraft still lands safely. Splitting this vertical error budget into a nominal position error budget and a budget for an undetected ionospheric error introduced by a single satellite affected by an ionospheric gradient allows the determination of a maximum residual position error $E_{v, \text { iono }}$ after applying the GBAS corrections. By taking into account the weighted pseudoinverse of the geometry matrix (often referred to as the $\mathrm{S}$ matrix) the largest residual pseudorange error $E_{r, \text { inono, } i}$ for a given satellite $i$ can be described as

$$
E_{r, \text { iono }, i} \leq \frac{E_{v, \text { iono }}}{\left|S_{\text {vert }, i}\right|}
$$

where $S_{\text {vert }, i}$ is the projection factor from the positioning algorithm which projects the pseudorange measurement into the vertical position. Attributing a missed detection probability of $10^{-9}$ to the monitor and assuming a Gaussian monitor noise the condition for the monitor can be described as

$$
I_{\text {test }, i} \leq \frac{E_{v, \text { iono }}}{\left|S_{\text {vert }, i}\right|}-6.1 \cdot \sigma_{\text {monitor }, i}
$$

The way the test statistic is formed yields a standard deviation for the monitor noise of

$$
\sigma_{\text {monitor }, i}=\frac{f_{L 5}^{2}}{f_{L 1}^{2}-f_{L 5}^{2}} \sqrt{\sigma_{g n d, L 1}^{2}+\sigma_{g n d, L 5}^{2}+\sigma_{\text {air }, L 1}^{2}+\sigma_{\text {air }, L 5}^{2}}
$$

where $f$ are the frequencies of the $\mathrm{L} 1$ and $\mathrm{L} 5$ signals and $\sigma_{g n d}$ and $\sigma_{\text {air }}$ the residual noise and multipath contained in the corrections from the ground and airborne measurements, respectively. Antenna and inter-frequency biases need to be further evaluated and might also need to be included in the monitor.

\section{DISCUSSION OF THE MONITOR}

The proposed monitor has certain properties worth discussing more in detail.

In the first place the assumption about the largest tolerable position error results from the touchdown requirements. It is therefore only valid for the last part of the approach until touchdown. It might be possible to relax the requirement for larger distances from the airport since less stringent requirements concerning the vertical position error may be necessary. Results in the next section, however, suggest that even with this conservative threshold problems would only occur in case of a minimum number of available satellites. Assuming a dual constellation scenario the number of satellites will almost always be large enough to guarantee a good performance. Another benefit of this monitor is that the threshold depends on the weight that is actually given to a certain satellite in the position solution.

The advantage of this monitor is its consideration of the actual weight a specific satellite is given in the position solution. In that way no stringent conservative assumptions have to be made but the current geometry is used to determine the monitoring requirements. Since medium elevation satellites typically have very small weights in the position solution the thresholds for these satellites can get arbitrarily large.

Figure 1 on the top plot shows the $s_{\text {vert }}$ values per satellite during one of our flight test. It can be seen that these projection factors decrease for elevations between about $35^{\circ}$ and $55^{\circ}$. The corresponding monitor thresholds in the lower figure increase accordingly. This reflects the situation that even if such a satellite would be affected by an ionospheric gradient it would hardly impact the position solution due to the low weight attributed to the satellite. 

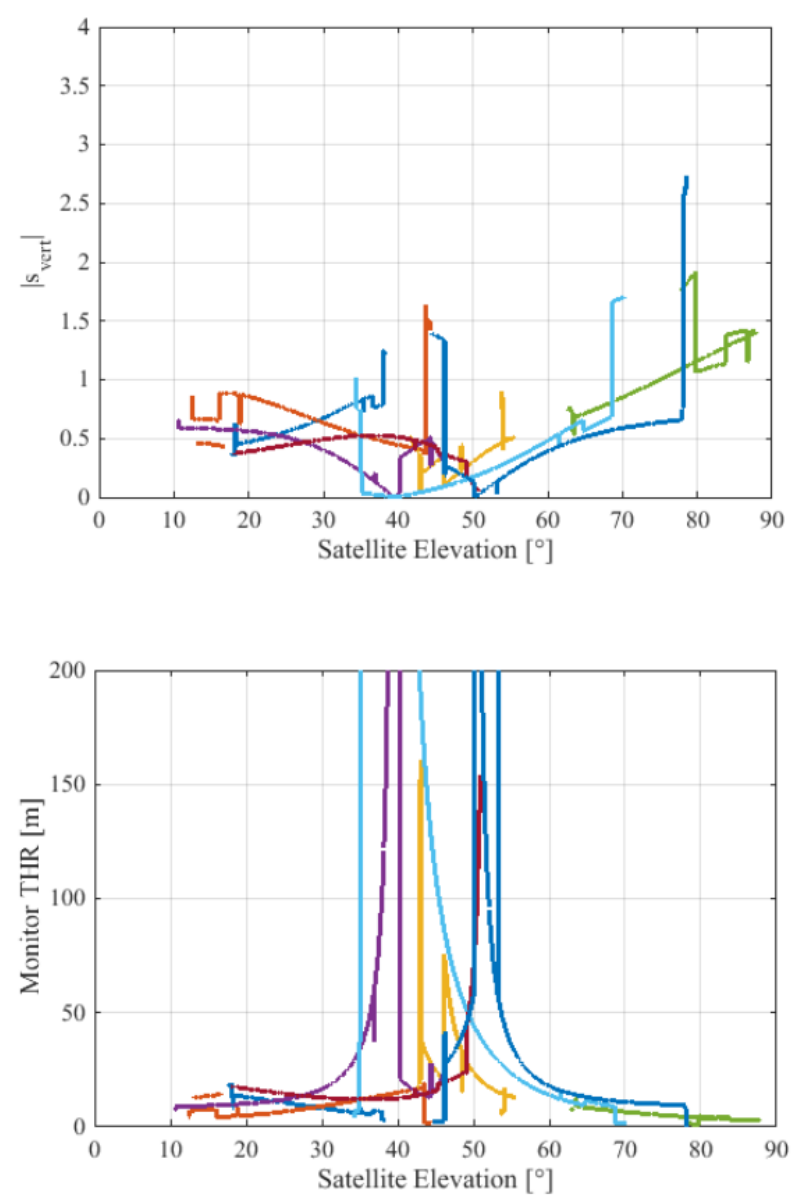

Figure 1 S_vert and monitor threshold as function of satellite elevation

In order to compute the monitoring threshold the noise in the monitor test statistic has to be accounted for and subtracted from the tolerable residual pseudorange error. While models for the L1 case exist and are used today, new models for the L5/E5a case have to be defined and validated. In previous work we already showed that the residual errors on the new signals are significantly lower than on the currently used ones [3],[4].

\section{EXPERIMENTAL VALIDATION WITH FLIGHT TEST DATA}

We now show the results of applying this monitor to flight data we collected during multi frequency multi constellation GBAS flight trials in March 2015 in Braunschweig in northern Germany [5]. The flight was a local flight during calm ionospheric conditions. Due to the sensitivity of the test statistic to the satellite geometry we first show the results for the L1/L2 dual frequency GPS case. During the flight we had 8 satellites in sight and available for navigation. This would be a representative case for a single constellation scenario. Moving to a dual frequency case the geometry would be even better and the monitoring thresholds even higher. Figure 2 shows an overview over the obtained thresholds and monitor test statistics. For better readability of the figures we limited the scale on the $y$-axis to a maximum of $20 \mathrm{~m}$. For the cases where the monitoring threshold is not visible in the figure it exceeds this limit.
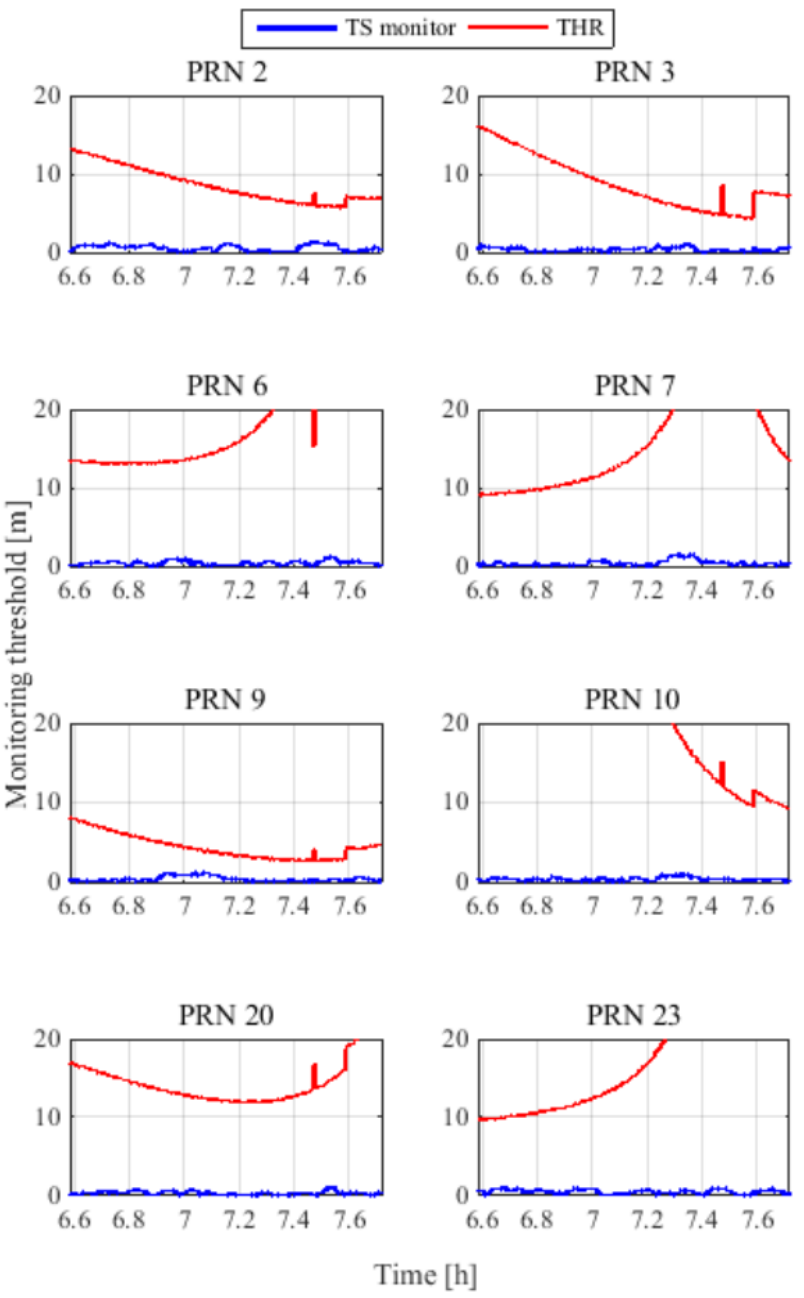

Figure 2 Monitoring thresholds (red) and test statistics (blue) during a test flight for the L1/L2 GPS case

At all times the test statistic remains well below the threshold as expected. The smallest monitoring threshold is encountered for PRN 9 which is a very high elevation satellite (green in Figure 3).

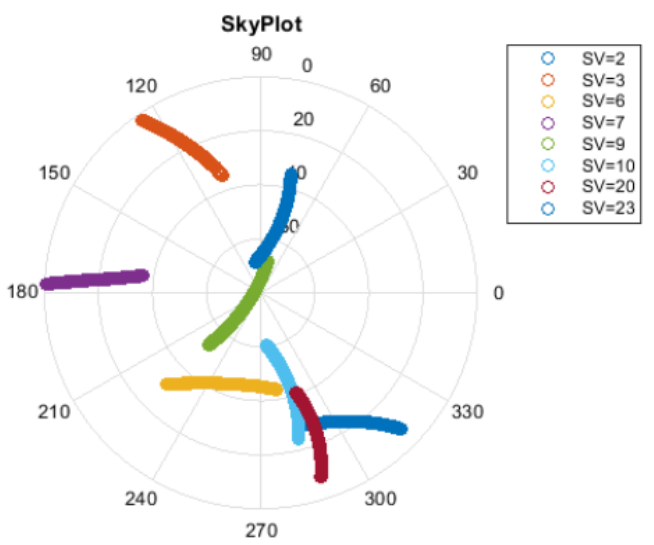

Figure 3 Skyplot of the GPS satellites during the flight test.

During the flight we also had up to 7 GPS Block IIF and Galileo satellites available that provide also signals in the 
L5/E5a frequency band. At about $7.4 \mathrm{~h}$ (as marked in Figure 4) we lost track of one satellite (PRN 81) reducing the number of satellites at that time to 5 . Since we were operating in a dual constellation mode this is actually the minimum number of satellites that can be used due to the five unknowns (positon and two clock offsets) in that case. Figure 5 shows the resulting performance for the monitor of PRN 6 at about $50^{\circ}$ elevation. While before the loss of PRN 81 it had a very low weight in the position solution it became a very important satellite after the loss leading to a significant decrease in the monitoring threshold very close to the value of the test statistic. However, even in that case with the lowest number of satellites the monitor did not trigger.

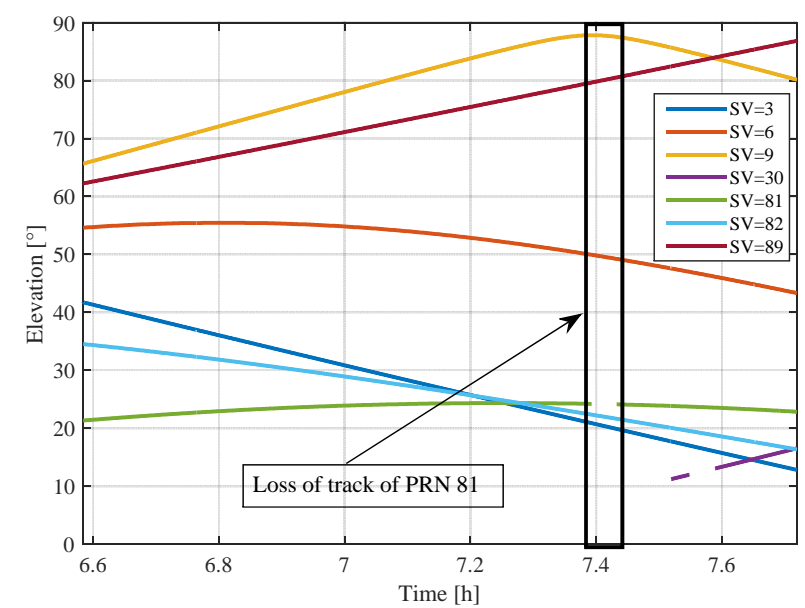

Figure 4 Elevation of the Block IIF (SV 3, 6, 9, 30) and Galileo satellites (SV 81, 82, 89) providing L5/E5a signals during the flight test.

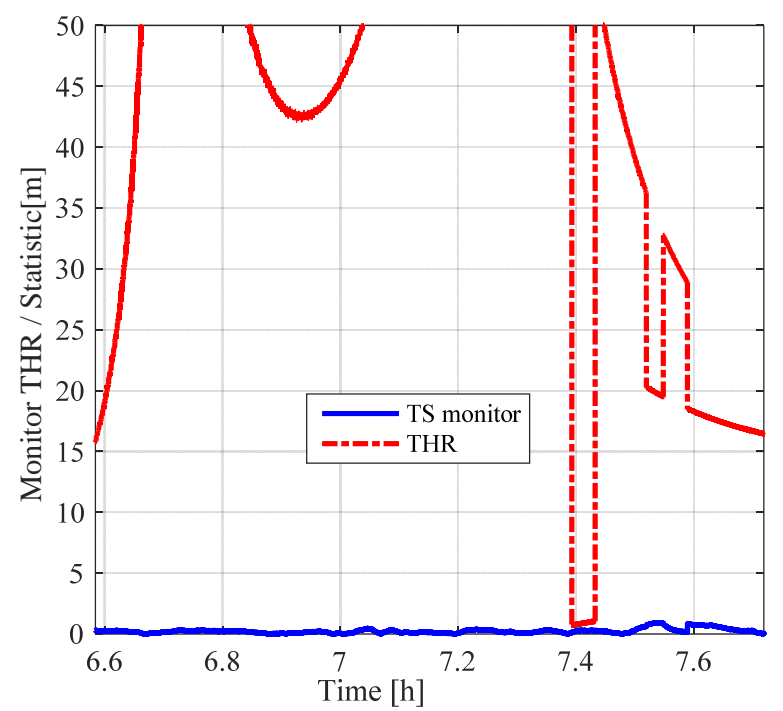

Figure 5 Monitor threshold and test statistic for PRN 6 for the dual frequency L1/L5 case.

The example shown here would be a very rare case in real operating conditions considering that an aircraft would be operating in a dual constellation mode with between 10 and 20 satellites and substantially stronger geometries [10].

\section{SIMULATION OF IONOSPHERIC ERRORS}

After looking at the nominal case we also want to evaluate the performance for an ionospheric gradient scenario. Since we only encountered calm conditions during our flights we injected an ionospheric ramp into the pseudorange and carrier phase measurements of PRN 2 and switched off all other monitors. The yellow shaded area in Figure 6 represents the time during which we simulated a gradient with a slope of $140 \mathrm{~mm} / \mathrm{km}$ and a relative speed w.r.t the aircraft of $200 \mathrm{~m} / \mathrm{s}$. The ramp increases to a maximum ionospheric delay of $15 \mathrm{~m}$ and remains at this value for the rest of the flight. The reaction of the monitor to the simulated gradient on PRN 2 is shown in the upper plot. It can be observed that the monitor starts reacting immediately with an initial increase of the test statistic. After reaching a first maximum of about $2 \mathrm{~m}$ it decreases again to zero and subsequently increases again. This effect is due to the reaction of the smoothing filter to the iono ramp. Initially more weight is given to the carrier phase measurements while with time the pseudorange measurements (affected by the ionosphere the same amount but with opposite sign than the carrier) become dominant. At about $6.9 \mathrm{~h}$ the threshold is exceeded which would lead to exclusion of the satellite or a switch to Ifree positioning. For illustration purposes we also show in blue the behavior of the monitor in case we did not exclude the satellite. Due to the lag of the smoothing filter the value of the test statistic keeps increasing for some time even after the maximum simulated iono delay is reached. After that it remains slightly below $15 \mathrm{~m}$.

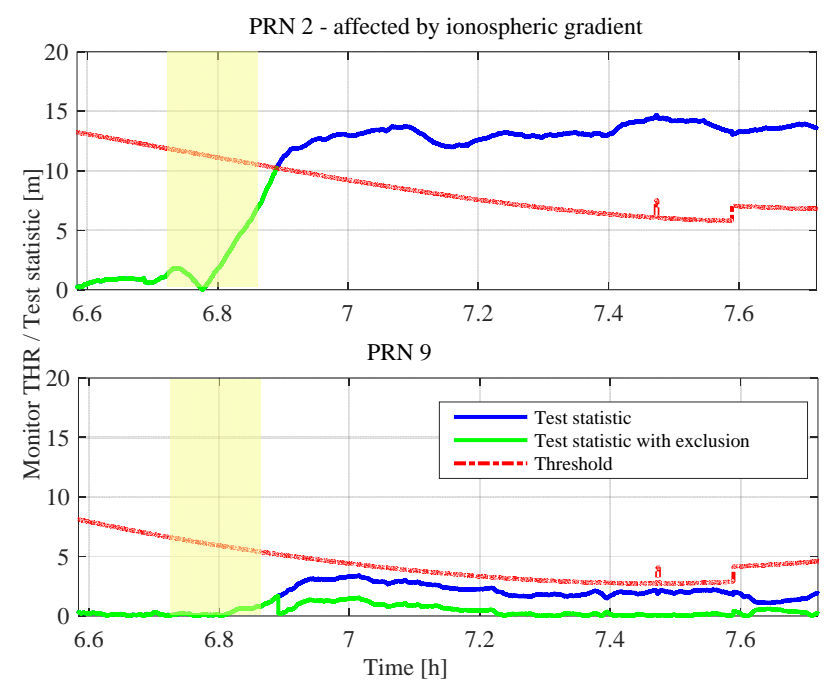

Figure 6 Reaction of the monitor to an ionospheric gradient for an affected satellite (top, PRN 2) and a not affected satellite (bottom, PRN 9). We show the reaction in case of satellite exclusion (green) and the effect on all other satellites without exclusion (blue). The period of the simulated ionospheric ramp is shaded in yellow.

In the lower plot we show the reaction of the monitor of another satellite, in this case PRN 9. The green curve 
shows the nominal case where the affected satellite (PRN 2 ) is excluded, the blue curve shows the monitor test statistic without exclusion. What can be seen is that if PRN 2 is not excluded from the set of satellites the test statistic of all other is affected as well due to the average removal process with the affected satellite as described in Equation (4).

\section{CONCLUSIONS}

Due to the better performance of single frequency positioning modes compared to the Ifree position solution in terms of protection levels and thus availability it is likely that these will be the primary modes for future GBAS architectures. However, when operating in a single frequency mode a monitoring for ionospheric gradients has to be performed. In this paper we showed a method of monitoring for ionospheric gradients in a dual frequency GBAS when positioning is based on single frequency measurements. In case the monitor detects an ionospheric gradient a satellite can either be excluded from the position solution or a switch to the Ifree processing mode can be triggered. By placing the burden of monitoring on the airborne side it is possible to use information about the actual satellite geometry and the corresponding projection factors to determine a threshold in real time. In that way unnecessarily conservative assumptions to cover all potentially possible cases can be avoided. Evaluation from flight test data showed that due to the realistic parameters good monitor performance can be achieved even in the case of very weak geometries with a minimum number of available satellites.

This work contributes to the research effort of developing future multi frequency and multi constellation GBAS which are expected to provide significantly improved robustness against different kinds of disturbances and yield improved availability in all regions of the world.

\section{ACKNOWLEDGMENTS}

The authors would like to thank their partners within the SESAR project 15.3.7, especially from the ground manufacturers Indra Navia and Thales Electronic System as well as Andreas Lipp from Eurocontrol for the fruitful discussion on this topic.

\section{REFERENCES}

[1] Gerbeth D., Circiu M.-S., Felux M., Caamano M, "Nominal Performance of Future Dual Frequency Dual Constellation GBAS," International Journal of Aerospace Engineering, vol. 2016, Article ID 6835282, 20 pages, 2016. doi:10.1155/2016/6835282

[2] Stanisak M., Lipp A., Feuerle T. "Possible VDB Formatting for Multi-Constellation / Multi-Frequency GBAS, Proc. Of the ION GNSS+ 2015, Tampa, FL, USA

[3] Circiu M.-S., Felux M., Belabbas B., Meurer M., Lee J., Pullen S, "Evaluation of GPS L5, Galileo E1 and Galileo E5a Performance in Flight Trials for Multi Frequency Multi Constellation GBAS” Proc. of the ION GNSS+ 2015, Tampa, FL, USA

[4] Circiu M.-S., Felux M., Thölert S., Antreich F., Vergara M., Sgammini M., Enneking C., Pullen S. "Evaluation of GPS L5 and Galileo E1 and E5a Performance for Future Multi Frequency and Multi Constellation GBAS” Proc. of the ION ITM 2015, Dana Point, CA, USA

[5] Felux M., Circiu M.-S., Gerbeth D., Caamano M., Stanisak M. (2016) "Ionospheric Monitoring in a Dual Frequency GBAS” Proceedings of the IEEE Aerospace Conference, Big Sky, MT, USA

[6] M.-S. Circiu, German Aerospace Center (DLR) \& RWTH Aachen University, Germany; M. Felux, D. Gerbeth, M. Caamaño “Assessment of Different Dual-frequency Dual-constellation GBAS Processing Modes Based on Flight Trials”, Proc. of the ION GNSS+ 2016, Portland, OR, USA

[7] Felux M., Circiu M.-S., Belabbas B., Meurer M., Stanisak M., Milner C., Jiang Y., Guilbert A., Lipp A. „Concept for a Dual Frequency Dual Constellation GBAS“, Proc. of the ION GNSS+ 2015, Tampa, FL, USA

[8] EASA 2003, Certification Specifications for All Weather Operations, (CS-AWO)

[9] FAA 1999, CRITERIA FOR APPROVAL OF CATEGORY III WEATHER MINIMA FOR TAKEOFF, LANDING, AND ROLLOUT, AC 12028D

[10] Gerbeth D., Felux M., Circiu M.-S., Caamano M., „Optimized Selection of Satellite Subsets for a Multi-Constellation GBAS“, Proc. of the ION ITM 2016, Monterey, CA, USA 